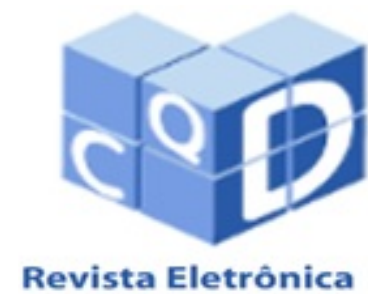

Paulista de Matemática

ISSN 2316-9664

Volume 13, dez. 2018

Fernando Neres de Oliveira

Universidade Federal Rural do

Semi-Árido - UFERSA

fernandoneres@ufersa.edu.br

\section{Uma prova elementar da irracionalidade de $\pi$}

An elementary proof of the irrationality of $\pi$

\section{Resumo}

Neste artigo, provamos de forma elementar a irracionalidade de $\pi^{2}$ (e, portanto, de $\pi$ ) usando o princípio da indução matemática, a técnica de integração por partes do cálculo elementar e alguns fatos básicos acerca de sequência infinita de números reais.

Palavras-chave: Irracionalidade de $\pi$. Irracionalidade de $\pi^{2}$. Princípio da indução matemática. Indução forte. Integração por partes. Sequência infinita de números reais.

\begin{abstract}
In this paper we prove in elementary form the irrationality of $\pi^{2}$ (and therefore, of $\pi$ ) using the principle of mathematical induction, the technique of integration by parts of the elementary calculus and some basic facts about infinite sequence of real numbers. Keywords: Irrationality of $\pi$. Irrationality of $\pi^{2}$. Principle of mathematical induction. Strong induction. Integration by parts. Infinite sequence of real numbers.
\end{abstract}




\section{Introdução}

O famoso número $\pi$, definido como a razão do comprimento de uma circunferência pelo seu diâmetro, é sem dúvida uma das mais importantes constantes matemáticas. Toda essa relevância é justificada pelas diversas conexões dessa constante com diferentes tópicos da matemática. Uma de suas principais características é o fato de $\pi$ ser um número irracional.

A irracionalidade de $\pi$ foi demonstrada pela primeira vez, ainda no século XVIII, pelo matemático francês Johann Heinrich Lambert. Na demonstração, Lambert (1767) obtém uma expansão da função tangente por frações contínuas e, a partir daí, ele consegue provar um resultado mais geral, a saber: "se $\alpha$ é um racional não nulo, então $\operatorname{tg} \alpha$ é irracional". Segue imediatamente deste fato que $\pi$ é irracional pois $\operatorname{tg}(\pi / 4)=1 \in \mathbb{Q}$.

$\mathrm{Na}$ literatura é possível encontrar diversas outras provas da irracionalidade de $\pi$. Niven (1947) demonstrou de forma simples a irracionalidade de $\pi$ usando o cálculo elementar. Breusch (1954) provou que $\pi$ é irracional usando a teoria de séries de potências. Em Niven (1956, p. 17), podemos achar um resultado análogo ao de Lambert para a função cosseno, a saber: "se $\beta$ é um racional não nulo, então $\cos \beta$ é irracional". Visto que $\cos (\pi)=-1 \in \mathbb{Q}$, então segue imediatamente deste fato que $\pi$ é irracional.

Hancl (1986) provou que $\pi^{4}$ é irracional, o que implica obviamente na irracionalidade de $\pi$ e $\pi^{2}$. Desbrow (1990) provou que não existe $\omega \in \mathbb{C}$ não nulo tal que $\omega^{2}$ e $\omega \operatorname{tgh}(\omega)$ sejam ambos racionais Gaussianos ${ }^{1}$. A partir, deste resultado fica fácil provar a irracionalidade de $\pi^{2}(\mathrm{e}$, portanto, de $\pi$ ), pois quando supomos o contrário obtemos que $(\pi i)^{2}$ e $(\pi i) \operatorname{tgh}(\pi i)$ são ambos racionais Gaussianos.

Estimulado por um problema proposto em Makarov et al (1992, p. 15), pretendemos, neste artigo, apresentar uma outra prova elementar da irracionalidade de $\pi$. Para isto, definiremos uma sequência de números reais que tem uma propriedade especial, a saber, o n-ésimo termo da sequência é a imagem de $\pi^{2}$ por um polinômio de grau $\leq n$ com coeficientes inteiros. Para provar que a referida sequência satisfaz tal propriedade, usaremos indução, integração por partes e alguns fatos básicos sobre sequências. A partir deste resultado, será possível provar a irracionalidade de $\pi^{2}$ (e, portanto, de $\pi$ ).

\section{O princípio da indução matemática}

O princípio da indução é uma valiosa técnica de demonstração matemática que nos permite demonstrar a validade de certas propriedades relativas a subconjuntos infinitos de $\mathbb{Z}$ (ou $\mathbb{N}$ ). A versão clássica e padrão deste princípio está contida no

Teorema 2.1 (Princípio da Indução Matemática (PIM 1)) Seja $S(n)$ uma sentença aberta relativa ao número natural $n$. Se

(i) S(1) é verdadeira, $e$

(ii) para todo $k \geq 1, S(k)$ verdadeira implicar $S(k+1)$ verdadeira, então a sentença $S(n)$ é verdadeira para todo $n \geq 1$.

\footnotetext{
${ }^{1}$ Um número complexo é um racional Gaussiano se ambas as suas partes real e imaginária são números racionais.
} 
O resultado acima pode ser provado diretamente a partir dos axiomas de Peano (veja Gunderson (2011, p. 24)). Chamamos a verificação de verdade para $S(1)$ de Base de Indução e a verificação de verdade da implicação " $S(k)$ verdadeira $\Rightarrow S(k+1)$ verdadeira" de Passo Indutivo. A suposição " $S(k)$ verdadeira" no passo indutivo é chamada Hipótese de Indução (HI).

Exemplo 2.1 Prove que a soma dos $n$ primeiros naturais ímpares é igual a $n^{2}$.

Solução: Seja $S(n)$ a seguinte sentença aberta relativa ao número natural $n$.

$$
S(n): 1+3+\cdots+(2 n-1)=n^{2} .
$$

Base de Indução $(n=1)$ : Temos que $1=1^{2}$. Logo, $S(1)$ é verdadeira.

Passo Indutivo $(S(k) \Rightarrow S(k+1))$ : Suponha que para todo $k \geq 1, S(k)$ é verdadeira, i.e.,

$$
1+3+\cdots+(2 k-1)=k^{2} \quad \text { (Hipótese de Indução). }
$$

Daí, obtemos que $1+3+\cdots+(2 k-1)+(2 k+1) \stackrel{\mathrm{HI}}{=} k^{2}+2 k+1=(k+1)^{2}$, i.e., $S(k+1)$ é verdadeira. Portanto, segue do princípio da indução que $S(n)$ é verdadeira para todo $n \geq 1$.

Exemplo 2.2 Prove que a soma dos n primeiros quadrados perfeitos é igual a $\frac{1}{6} n(n+1)(2 n+1)$.

Solução: Seja $S(n)$ a seguinte sentença aberta relativa ao número natural $n$.

$$
S(n): 1^{2}+2^{2}+\cdots+n^{2}=\frac{1}{6} n(n+1)(2 n+1) .
$$

Base de Indução $(n=1)$ : Temos que $1^{2}=1=\frac{1}{6} \cdot 1 \cdot(1+1) \cdot(2 \cdot 1+1)$. Logo, $S(1)$ é verdadeira. Passo Indutivo $(S(k) \Rightarrow S(k+1))$ : Suponha que para todo $k \geq 1, S(k)$ é verdadeira, i.e.,

$$
1^{2}+2^{2}+\cdots+k^{2}=\frac{1}{6} k(k+1)(2 k+1) \quad \text { (Hipótese de Indução). }
$$

Daí, obtemos que,

$$
\begin{aligned}
1^{2}+2^{2}+\cdots+k^{2}+(k+1)^{2} & \stackrel{\text { HI }}{=} \frac{1}{6} k(k+1)(2 k+1)+(k+1)^{2} \\
& =(k+1)\left[\frac{1}{6} k(2 k+1)+(k+1)\right] \\
& =(k+1) \frac{(k+2)(2 k+3)}{6} \\
& =\frac{1}{6}(k+1)[(k+1)+1][2(k+1)+1],
\end{aligned}
$$

i.e., $S(k+1)$ é verdadeira. Portanto, segue do princípio da indução que $S(n)$ é verdadeira para todo $n \geq 1$.

Exemplo 2.3 (Desigualdade de Bernoulli) Seja x um número real não nulo, com $x>-1$. Prove que para todo $n \geq 1$,

$$
(1+x)^{n} \geq 1+n x
$$


Solução: Seja $S(n)$ a seguinte sentença aberta relativa ao número natural $n$.

$$
S(n):(1+x)^{n} \geq 1+n x .
$$

Base de Indução $(n=1)$ : Temos que $(1+x)^{1}=1+x=1+1 \cdot x$. Logo, $S(1)$ é verdadeira. Passo Indutivo $(S(k) \Rightarrow S(k+1))$ : Suponha que para todo $k \geq 1, S(k)$ é verdadeira, i.e.,

$$
(1+x)^{k} \geq 1+k x \quad \text { (Hipótese de Indução). }
$$

Como $1+x>0$, então segue que,

$$
(1+x)^{k+1}=(1+x)^{k}(1+x) \stackrel{\mathrm{HI}}{\geq}(1+k x)(1+x)=1+x+k x+k x^{2} \underset{x \neq 0}{>} 1+x+k x=1+(k+1) x,
$$

i.e., $S(k+1)$ é verdadeira. Portanto, segue do princípio da indução que $S(n)$ é verdadeira para todo $n \geq 1$.

Vale ressaltar que a base de indução não precisa ser considerada necessariamente em $n=1$. O princípio continua válido se a indução começar a partir de qualquer inteiro. Uma forma mais geral deste princípio está contida no

Teorema 2.2 (Princípio da Indução Matemática (PIM 2)) Sejam S(n) uma sentença aberta relativa ao número natural n e $k \in \mathbb{Z}$ fixado. Se

(i) $S(k)$ é verdadeira, $e$

(ii) para todo $m \geq k, S(m)$ verdadeira implicar $S(m+1)$ verdadeira,

então a sentença $S(n)$ é verdadeira para todo $n \geq k$.

Demonstração: Veja Gunderson (2011, p. 36).

Às vezes, nos deparamos com algumas situações em que a suposição de verdade para $S(k)$ $(k \geq 1)$ não é suficiente para provar a validade de $S(k+1)$. Neste caso, é necessário fortalecer um pouco mais a hipótese indutiva, supondo que a sentença $S(n)$ seja verdadeira para outros inteiros $n$ menores do que $k$. Esta situação é contemplada pela seguinte variante do princípio da indução.

Teorema 2.3 (Princípio da Indução Forte) Seja $S(n)$ uma sentença aberta relativa ao número natural n. Se

(i) $S(k)$ é verdadeira, $e$

(ii) para todo $m \geq k, S(k), S(k+1), \ldots, S(m)$ verdadeiras implicar $S(m+1)$ verdadeira, então a sentença $S(n)$ é verdadeira para todo $n \geq k$.

Demonstração: Veja Gunderson (2011, p. 37).

As versões apresentadas nos Teoremas 2.2 e 2.3 são, na verdade, versões de um mesmo princípio. É possível provar que tais versões são equivalentes (veja Gunderson (2011, p. 37)). 
Exemplo 2.4 Prove que qualquer inteiro positivo $n \geq 2$ é um produto de primos.

Solução: Seja $S(n)$ a seguinte sentença aberta relativa ao número natural $n$.

$$
S(n): n \text { é um produto de primos. }
$$

Base de Indução $(n=2)$ : Temos que 2 é primo. Logo, $S(2)$ é verdadeira.

Passo Indutivo: Seja $m \geq 2$ um inteiro fixado. Suponha que $S(t)$ é verdadeira para todo inteiro $\mathrm{t}$ satisfazendo $2 \leq t \leq m$ (Hipótese de Indução). Se $m+1$ é primo, então $S(m+1)$ é verdadeira. Se $m+1$ não é primo, então existem inteiros $2 \leq b \leq m$ e $2 \leq c \leq m$ tais que $m+1=b c$. Daí, segue da hipótese de indução que $S(b)$ e $S(c)$ são ambas verdadeiras, i.e., $b$ é um produto de primos e $c$ é um produto de primos. Logo, $b c$ é um produto de primos, i.e., $S(m+1)$ é verdadeira. Em qualquer caso, tem-se que $S(m+1)$ é verdadeira. Portanto, segue do princípio da indução (forte) que $S(n)$ é verdadeira para todo $n \geq 2$.

Exemplo 2.5 Para todo inteiro $r \geq 1$, prove que se $r^{2}=$ st e s e t são relativamente primos, então s e t são ambos quadrados.

Solução: Seja $S(r)$ a seguinte sentença aberta relativa ao número natural $r$.

$$
S(r): r^{2}=s t \text { e } m d c(s, t)=1 \Longrightarrow s \text { e } t \text { são ambos quadrados. }
$$

Base de Indução $(r=1)$ : Neste caso a única forma de decompor $1^{2}$ como produto de inteiros relativamente primos é $1^{2}=1 \cdot 1$. Como os fatores 1 e 1 são ambos quadrados, então, $S(1)$ é verdadeira.

Passo Indutivo: Seja $m \geq 1$ um inteiro fixado. Suponha que $S(j)$ é verdadeira para todo inteiro j satisfazendo $1 \leq j \leq m$ (Hipótese de Indução). Sejam $s$ e $t$ inteiros positivos tais que $(m+1)^{2}=s t$ e $m d c(s, t)=1$. Seja $p$ um divisor primo de $m+1$. Então, $\frac{m+1}{p}$ e $\frac{(m+1)^{2}}{p^{2}}$ são ambos inteiros. Além disso, como $p$ é primo e $m d c(s, t)=1$ então $p$ divide exatamente um dos inteiros $s$ ou $t$. Sem perda de generalidade, vamos supor que $p \mid s$, mas $p \nmid t$. Nesse caso, como $p^{2} \mid(m+1)^{2}$, então segue que $p^{2} \mid s$, i.e., $s=p^{2} x, \operatorname{com} x \in \mathbb{N}$. Agora, perceba então que,

$$
\left(\frac{m+1}{p}\right)^{2}=\frac{(m+1)^{2}}{p^{2}}=\frac{s t}{p^{2}}=\frac{p^{2} x t}{p^{2}}=x t,
$$

onde $m d c(x, t)=1$ (caso contrário, teríamos que $m d c(s, t) \neq 1$ ). Daí, como $\frac{m+1}{p}$ é um inteiro satisfazendo $1 \leq \frac{m+1}{p} \leq m$, então segue da hipótese de indução que $x$ e $t$ são quadrados. Mas, isso implica então que $s$ e $t$ são quadrados. Ou seja, $S(m+1)$ é verdadeira. Portanto, segue do princípio da indução (forte) que $S(r)$ é verdadeira para todo inteiro $r \geq 1$.

Exemplo 2.6 A sequência de Fibonacci $F_{n}$ é a sequência definida recursivamente por $F_{0}=0$, $F_{1}=1$ e $F_{n}=F_{n-1}+F_{n-2}$, para todo $n \geq 2$. Prove que para todo $n \geq 0$,

$$
F_{n}=\frac{\alpha^{n}-\beta^{n}}{\alpha-\beta}
$$

onde $\alpha=\frac{1+\sqrt{5}}{2}$ e $\beta=\frac{1-\sqrt{5}}{2}$ são as raízes da equação $x^{2}=x+1$. 
Solução: Seja $S(n)$ a seguinte sentença aberta relativa ao inteiro não negativo $n$.

$$
S(n): F_{n}=\frac{\alpha^{n}-\beta^{n}}{\alpha-\beta} \text { onde } \alpha=\frac{1+\sqrt{5}}{2} \text { e } \beta=\frac{1-\sqrt{5}}{2} .
$$

Base de Indução $(n=0,1)$ : Temos de $S(0)$ e $S(1)$ que $F_{0}=\frac{\alpha^{0}-\beta^{0}}{\alpha-\beta}=0$ e $F_{1}=\frac{\alpha^{1}-\beta^{1}}{\alpha-\beta}=1$. Logo, $S(0)$ e $S(1)$ são ambas verdadeiras.

Passo Indutivo: Seja $m \geq 1$ um inteiro fixado. Suponha que $S(t)$ é verdadeira para todo inteiro $t$ satisfazendo $0 \leq t \leq m$ (Hipótese de Indução). Daí, obtemos que,

$F_{m+1}=F_{m-1}+F_{m} \stackrel{\mathrm{HI}}{=} \frac{\alpha^{m-1}-\beta^{m-1}}{\alpha-\beta}+\frac{\alpha^{m}-\beta^{m}}{\alpha-\beta}=\frac{\alpha^{m-1}(1+\alpha)-\beta^{m-1}(1+\beta)}{\alpha-\beta}=\frac{\alpha^{m+1}-\beta^{m+1}}{\alpha-\beta}$,

i.e., $S(m+1)$ é verdadeira. Portanto, segue do princípio da indução (forte) que $S(n)$ é verdadeira para todo $n \geq 0$.

\section{A técnica da integração por partes}

Sabemos do Teorema Fundamental do Cálculo $^{2}$ (TFC) que a integral de uma função contínua $f$ em $[a, b]$ pode ser facilmente calculada a partir de uma primitiva de $f$ em $[a, b]$. Porém, a tarefa de achar uma primitiva para uma dada função $f$ não é, em geral, trivial. Há certas classes de funções que para serem integradas necessitam de técnicas específicas de integração. Por exemplo, para o cálculo da integral indefinida

$$
\int e^{x} \cos x d x
$$

necessitamos de uma técnica chamada Integração por Partes, a qual passaremos agora a descrever. Suponha que $f$ e $g$ são funções deriváveis de $x$. A regra do produto nos diz então que

$$
\frac{d}{d x}[f(x) g(x)]=f^{\prime}(x) g(x)+f(x) g^{\prime}(x),
$$

em termos de integrais indefinidas, temos então que,

$$
\int \frac{d}{d x}[f(x) g(x)] d x=\int f^{\prime}(x) g(x) d x+\int f(x) g^{\prime}(x) d x
$$

ou seja,

$$
\int f(x) g^{\prime}(x) d x=f(x) g(x)-\int f^{\prime}(x) g(x) d x
$$

A fórmula (1) é chamada fórmula da integração por partes. Essa fórmula é mais facilmente lembrada quando escrita na forma diferencial, a saber: se $u=f(x)$ e $v=g(x)$, então as diferenciais de $u$ e $v$ são $d u=f^{\prime}(x) d x$ e $d v=g^{\prime}(x) d x$. Assim, a fórmula (1) pode ser reescrita da seguinte forma

$$
\int u d v=u v-\int v d u
$$

${ }^{2}$ Se $f$ é contínua em $[a, b]$ e $F$ é uma primitiva de $f$ em $[a, b]$ então $\int_{a}^{b} f(x) d x=F(b)-F(a)$. 
Exemplo 3.1 Calcule $\int x(\ln x)^{2} d x$.

Solução: Fazendo $u=(\ln x)^{2}$ e $d v=x d x$, obtemos que, $d u=\frac{2 \ln x}{x} d x$ e $v=\frac{x^{2}}{2}$. Daí, segue da fórmula (2) que

$$
\int x(\ln x)^{2} d x=\int \underbrace{(\ln x)^{2}}_{u} \underbrace{x d x}_{d v}=u v-\int v d u=\frac{x^{2}(\ln x)^{2}}{2}-\int(\ln x) x d x .
$$

Para calcular a integral de $x \ln x$ aplicamos novamente a técnica da integração por partes. Faça $z=\ln x$ e $d w=x d x$, então, $d z=\frac{1}{x} d x$ e $w=\frac{x^{2}}{2}$. Assim, segue da fórmula (2) que

$\int x(\ln x)^{2} d x=\frac{x^{2}(\ln x)^{2}}{2}-\int \underbrace{(\ln x)}_{z} \underbrace{x d x}_{d w}=\frac{x^{2}(\ln x)^{2}}{2}-z w+\int w d z=\frac{x^{2}(\ln x)^{2}}{2}-\frac{x^{2} \ln x}{2}+\frac{x^{2}}{4}+C$.

Exemplo 3.2 Calcule $\int e^{x} \cos x d x$.

Solução: Fazendo $u=\cos x$ e $d v=e^{x} d x$, obtemos que, $d u=-\operatorname{sen} x d x$ e $v=e^{x}$. Daí, segue da fórmula (2) que

$$
\int e^{x} \cos x d x=\int \underbrace{\cos x}_{u} \underbrace{e^{x} d x}_{d v}=u v-\int v d u=e^{x} \cos x+\int e^{x} \operatorname{sen} x d x .
$$

Usaremos novamente a técnica da integração por partes para calcular a integral de $e^{x} \operatorname{sen} x$. Faça $z=\operatorname{sen} x$ e $d w=e^{x} d x$, então, $d z=\cos x d x$ e $w=e^{x}$. Assim, segue da fórmula (2) que

$\int e^{x} \cos x d x=e^{x} \cos x+\int \underbrace{\operatorname{sen} x}_{z} \underbrace{e^{x} d x}_{d w}=e^{x} \cos x+z w-\int w d z=e^{x} \cos x+e^{x} \operatorname{sen} x-\int e^{x} \cos x d x$.

Portanto, $\int e^{x} \cos x d x=\frac{e^{x}(\operatorname{sen} x+\cos x)}{2}+C$.

A técnica da integração por partes também pode ser aplicada às integrais definidas. Assim como tivemos para integrais indefinidas, haverá também uma fórmula da integração por partes para integrais definidas. Este fato está contido na

Proposição 3.1 Se as funções $f, g:[a, b] \rightarrow \mathbb{R}$ têm derivadas contínuas então,

$$
\int_{a}^{b} f(x) g^{\prime}(x) d x=[f(x) g(x)]_{a}^{b}-\int_{a}^{b} f^{\prime}(x) g(x) d x .
$$

Demonstração: Temos que $f(x) g(x)$ é uma primitiva de $f(x) g^{\prime}(x)+f^{\prime}(x) g(x)$ em $[a, b]$. Daí, segue do TFC que

$$
\int_{a}^{b} f(x) g^{\prime}(x) d x+\int_{a}^{b} f^{\prime}(x) g(x) d x=\int_{a}^{b}\left[f(x) g^{\prime}(x)+f^{\prime}(x) g(x)\right] d x=[f(x) g(x)]_{a}^{b} .
$$


Exemplo 3.3 Calcule $\int_{1}^{e} \frac{\ln x}{x^{2}} d x$.

Solução: Sejam $u=\ln x$ e $d v=x^{-2} d x$. Então, $d u=x^{-1} d x$ e $v=-x^{-1}$. Daí, segue da Proposição 3.1 que

$\int_{1}^{e} \frac{\ln x}{x^{2}} d x=\int_{1}^{e} \underbrace{(\ln x)}_{u} \underbrace{x^{-2} d x}_{d v}=[u v]_{1}^{e}-\int_{1}^{e} v d u=\left[-x^{-1} \ln x\right]_{1}^{e}+\int_{1}^{e} x^{-2} d x=-2 e^{-1}+1=1-\frac{2}{e}$.

Exemplo 3.4 Calcule $\int_{0}^{\pi} e^{x} \operatorname{sen} x d x$.

Solução: Sejam $u=\operatorname{sen} x$ e $d v=e^{x} d x$. Então, $d u=\cos x d x$ e $v=e^{x}$. Daí, segue da Proposição 3.1 que

$\int_{0}^{\pi} e^{x} \operatorname{sen} x d x=\int_{0}^{\pi} \underbrace{(\operatorname{sen} x)}_{u} \underbrace{e^{x} d x}_{d v}=[u v]_{0}^{\pi}-\int_{0}^{\pi} v d u=\left[e^{x} \operatorname{sen} x\right]_{0}^{\pi}-\int_{0}^{\pi} e^{x} \cos x d x=-\int_{0}^{\pi} e^{x} \cos x d x$

No Exemplo 3.2 já determinamos todas as primitivas da função $e^{x} \cos x$. Então, segue do TFC que

$$
\int_{0}^{\pi} e^{x} \operatorname{sen} x d x=-\int_{0}^{\pi} e^{x} \cos x d x=-\left[\left(\frac{e^{x}(\operatorname{sen} x+\cos x)}{2}\right)\right]_{0}^{\pi}=\frac{e^{\pi}+1}{2} .
$$

\section{Sequência infinita de números reais}

No que segue, definiremos sequência de números reais e apresentaremos alguns resultados básicos que serão úteis na próxima seção.

Definição 4.1 Sejam $k$ um inteiro fixado e $K$ o conjunto de todos os inteiros maiores ou iguais a k. Uma sequência infinita (ou mais brevemente, uma sequência) de números reais é qualquer função $s: K \rightarrow \mathbb{R}$. As imagens dos elementos de $K$ são chamadas os termos da sequência.

Assim, dada uma sequência $s: K \rightarrow \mathbb{R}$, nós usualmente escrevemos $s_{n}$ em vez de $s(n)$. Além disso, denotamos a sequência por qualquer uma das seguintes notações: $\left\{s_{n}\right\}_{n \in K},\left\{s_{n}\right\}_{n \geq k}$ ou simplesmente $\left\{s_{n}\right\}$, quando o conjunto $K$ for claramente identificado.

Exemplo 4.1 A sequência dada por $\left\{(-1)^{n+1}\right\}_{n \geq 1}$ é $1,-1,1,-1,1,-1, \ldots$

Exemplo 4.2 A sequência dada por $\left\{\frac{1}{n^{2}+1}\right\}_{n \geq 0}$ é $1, \frac{1}{2}, \frac{1}{5}, \frac{1}{10}, \frac{1}{17}, \ldots$

Exemplo 4.3 A sequência dada por $\left\{(1+1 / n)^{n}\right\}_{n \geq 1}$ é $2, \frac{9}{4}, \frac{64}{27}, \frac{625}{256}, \ldots$ 
Definição 4.2 Sejam $\left\{s_{n}\right\}$ uma sequência de números reais e L um número real. Diremos que $\left\{s_{n}\right\}$ converge para $L$, e que Lé o limite de $\left\{s_{n}\right\}$, se para cada $\varepsilon>0$ existir um inteiro $n_{0}$ tal que

$$
\left|s_{n}-L\right|<\varepsilon
$$

sempre que $n \geq n_{0}$. Neste caso, dizemos que a sequência $\left\{s_{n}\right\}$ é convergente, e escrevemos

$$
\lim _{n \rightarrow \infty} s_{n}=L
$$

Caso contrário, a sequência $\left\{s_{n}\right\}$ é dita divergente.

Exemplo 4.4 A sequência constante $\{c\}$ é convergente e seu limite é c.

Exemplo 4.5 A sequência $\left\{(-1)^{n+1}\right\}_{n \geq 1}$ é divergente.

Exemplo 4.6 A sequência $\{\pi+1 / n\}_{n \geq 1}$ é convergente e seu limite é $\pi$.

Lema 4.1 Se $\left\{x_{n}\right\}_{n \geq 0}$ é uma sequência convergente de inteiros positivos, então $\lim _{n \rightarrow \infty} x_{n} \neq 0$.

Demonstração: Suponha que $\lim _{n \rightarrow \infty} x_{n}=0$. Seja $\varepsilon$ um número real tal que $0<\varepsilon<1$. Daí, segue da definição de limite para sequências que, existe $n_{0} \in \mathbb{N} \cup\{0\}$ tal que $0<x_{n}<\varepsilon<1$ para todo $n \geq n_{0}$ (contradição!).

Exemplo 4.7 $x_{n}=\left\{\begin{array}{ll}n^{2}+82, & \text { se } n<44 \\ 2018, & \text { se } n \geq 44\end{array}\right.$ é uma sequência convergente de inteiros positivos tal que $\lim _{n \rightarrow \infty} x_{n}=2018 \neq 0$.

Lema 4.2 Sejam $\left\{x_{n}\right\}_{n \geq 0}$ uma sequência de números reais e $k_{0}$ um inteiro não negativo. Então, $\lim _{n \rightarrow \infty} x_{k_{0}+n}=L$ se, e somente se, $\lim _{n \rightarrow \infty} x_{n}=L$.

Demonstração: $(\Rightarrow)$ Seja $\varepsilon>0$ um número real. Como $\lim _{n \rightarrow \infty} x_{k_{0}+n}=L$ então existe $n_{0} \in \mathbb{N} \cup\{0\}$ tal que $\left|x_{k_{0}+n}-L\right|<\varepsilon$ sempre que $n \geq n_{0}$. Daí, para cada inteiro $n \geq k_{0}+n_{0}$, tem-se que, $\left|x_{n}-L\right|<\varepsilon$. Portanto, $\lim _{n \rightarrow \infty} x_{n}=L$.

$(\Leftarrow)$ Seja $\varepsilon>0$ um número real. Como $\lim _{n \rightarrow \infty} x_{n}=L$ então existe $n_{0} \in \mathbb{N} \cup\{0\}$ tal que $\left|x_{n}-L\right|<\varepsilon$ sempre que $n \geq n_{0}$. Sendo $k_{0}$ inteiro não negativo, então $k_{0}+n \geq n_{0}$ sempre que $n \geq n_{0}$. Daí, para cada inteiro $n \geq n_{0}$, tem-se que, $\left|x_{k_{0}+n}-L\right|<\varepsilon$. Portanto, $\lim _{n \rightarrow \infty} x_{k_{0}+n}=L$.

Proposição 4.1 Se $\lim _{n \rightarrow \infty}\left|x_{n}\right|=0$, então $\lim _{n \rightarrow \infty} x_{n}=0$.

Demonstração: Seja $\varepsilon>0$ um número real. Como $\lim _{n \rightarrow \infty}\left|x_{n}\right|=0$, então existe um inteiro não negativo $n_{0}$ tal que ||$x_{n}|-0|<\varepsilon$ sempre que $n \geq n_{0}$. Ou seja, existe um inteiro não negativo $n_{0}$ tal que $\left|x_{n}\right|<\varepsilon$ sempre que $n \geq n_{0}$. Portanto, $\lim _{n \rightarrow \infty} x_{n}=0$.

Teorema 4.1 (Teorema do Sanduíche) Se $\lim _{n \rightarrow \infty} x_{n}=\lim _{n \rightarrow \infty} y_{n}=L$ e $x_{n} \leq z_{n} \leq y_{n}$ para todo $n$ suficientemente grande, então $\lim _{n \rightarrow \infty} z_{n}=L$ 
Demonstração: Seja $\varepsilon>0$ um número real. Segue da hipótese que existem $n_{1}, n_{2}, n_{3} \in \mathbb{N} \cup\{0\}$ tais que

$$
\begin{aligned}
& n \geq n_{1} \Rightarrow L-\varepsilon<x_{n}<L+\varepsilon \\
& n \geq n_{2} \Rightarrow L-\varepsilon<y_{n}<L+\varepsilon \\
& n \geq n_{3} \Rightarrow x_{n} \leq z_{n} \leq y_{n} .
\end{aligned}
$$

Tome $n_{0}=\max \left\{n_{1}, n_{2}, n_{3}\right\}$. Daí, para cada inteiro $n \geq n_{0}$, tem-se que,

$$
L-\varepsilon<x_{n} \leq z_{n} \leq y_{n}<L+\varepsilon .
$$

Portanto, $\lim _{n \rightarrow \infty} z_{n}=L$.

Proposição 4.2 Se $0<s<1$, então $\lim _{n \rightarrow \infty} s^{n}=0$.

Demonstração: Temos que $r=\frac{1-s}{s}>0$. Logo, $s=\frac{1}{1+r}$. Usando agora a desigualdade de Bernoulli (Exemplo 2.3), obtém-se que,

$$
0<s^{n}=\frac{1}{(1+r)^{n}} \leq \frac{1}{1+n r}<\frac{1}{n r},
$$

para todo $n \geq 1$. Daí, como $\lim _{n \rightarrow \infty} \frac{1}{n r}=0$, então segue do Teorema 4.1 que $\lim _{n \rightarrow \infty} s^{n}=0$.

Proposição 4.3 Seja $\left\{x_{n}\right\}_{n \geq 0}$ uma sequência de números reais não nulos e suponha que

$$
\lim _{n \rightarrow \infty}\left|\frac{x_{n+1}}{x_{n}}\right|=L<1
$$

Então,

$$
\lim _{n \rightarrow \infty} x_{n}=0
$$

Demonstração: Temos que o número $\varepsilon=(1-L) / 2>0$, pois $L<1$. Como $\lim _{n \rightarrow \infty}\left|\frac{x_{n+1}}{x_{n}}\right|=L$, então segue da definição de limite para sequências que, existe $k_{0} \in \mathbb{N} \cup\{0\}$ tal que

$$
0<\frac{\left|x_{n+1}\right|}{\left|x_{n}\right|}<L+\varepsilon=\frac{L+1}{2}
$$

sempre que $n \geq k_{0}$. Daí, segue do princípio da indução que ${ }^{3}$

$$
0<\left|x_{k_{0}+n}\right|<\left(\frac{L+1}{2}\right)^{n}\left|x_{k_{0}}\right|
$$

${ }^{3}$ (i) Para $n=1$, tem-se que, $\left|x_{k_{0}+1}\right|<\left(\frac{L+1}{2}\right)\left|x_{k_{0}}\right|$, o que é verdadeiro; (ii) Suponha que a desigualdade é verdadeira para um inteiro $n=m \geq 1$, i.e., $\left|x_{k_{0}+m}\right|<\left(\frac{L+1}{2}\right)^{m}\left|x_{k_{0}}\right|$ (Hipótese de Indução (HI)). Daí, obtém-se que, $\left|x_{k_{0}+(m+1)}\right|=\left|x_{\left.\left(k_{0}+m\right)+1\right)}\right| \underbrace{<}_{(3)}\left(\frac{L+1}{2}\right)\left|x_{k_{0}+m}\right|<\frac{L+1}{2})^{m+1}\left|x_{k_{0}}\right|$, i.e., a desigualdade é verdadeira para o inteiro $n=m+1$. Portanto, segue do princípio da indução que $0<\left|x_{k_{0}+n}\right|<\left(\frac{L+1}{2}\right)^{n}\left|x_{k_{0}}\right|$ para todo $n \in \mathbb{N}$. 
para todo $n \in \mathbb{N}$. Por outro lado, como $0<\frac{L+1}{2}<1$, então segue da Proposição 4.2 que

$$
\lim _{n \rightarrow \infty}\left(\frac{L+1}{2}\right)^{n}\left|x_{k_{0}}\right|=0
$$

Usando agora o Teorema 4.1 obtemos que $\lim _{n \rightarrow \infty}\left|x_{k_{0}+n}\right|=0$. Assim, concluímos da Proposição 4.1 e do Lema 4.2 que $\lim _{n \rightarrow \infty} x_{n}=0$.

Exemplo 4.8 $\lim _{n \rightarrow \infty} \frac{a^{n}}{n !}=0$, para todo $a \in \mathbb{R}$.

Solução: Considere a sequência $\left\{x_{n}\right\}_{n \geq 1}$, definida por $x_{n}=\frac{a^{n}}{n !}$, onde $a$ é um número real fixado. É óbvio que, $\lim _{n \rightarrow \infty} \frac{0^{n}}{n !}=0$. Suponha agora que $a \neq 0$. Então,

$$
\left|\frac{x_{n+1}}{x_{n}}\right|=\left|\frac{a^{n+1}}{(n+1) !} \cdot \frac{n !}{a^{n}}\right|=\left|\frac{a}{n+1}\right|=\frac{|a|}{n+1}
$$

e

$$
\lim _{n \rightarrow \infty}\left|\frac{x_{n+1}}{x_{n}}\right|=\lim _{n \rightarrow \infty} \frac{|a|}{n+1}=0<1 .
$$

Portanto, segue da Proposição 4.3 que $\lim _{n \rightarrow \infty} x_{n}=0$.

\section{A irracionalidade de $\pi$}

Considere a sequência $\left\{I_{n}\right\}_{n \geq 0}$ definida por

$$
I_{n}=\frac{1}{n !} \int_{-\pi / 2}^{\pi / 2}\left(\frac{\pi^{2}}{4}-t^{2}\right)^{n} \cos t d t
$$

Proposição $5.1 I_{n}>0$ para todo inteiro $n$ não negativo.

Demonstração: Seja $n$ um inteiro não negativo fixado. Como $f(t)=\left(\frac{\pi^{2}}{4}-t^{2}\right)^{n} \cos t$ é uma função par então,

$$
I_{n}=\frac{1}{n !} \int_{-\pi / 2}^{\pi / 2}\left(\frac{\pi^{2}}{4}-t^{2}\right)^{n} \cos t d t=\frac{2}{n !} \int_{0}^{\pi / 2}\left(\frac{\pi^{2}}{4}-t^{2}\right)^{n} \cos t d t .
$$

Mas, $\frac{\pi^{2}}{4}-t^{2} \geq 0$ e cost $\geq 0$ no intervalo $[0, \pi / 2]$. Logo, $f(t)=\left(\frac{\pi^{2}}{4}-t^{2}\right)^{n} \cos t \geq 0$, para todo $t \in[0, \pi / 2]$. Daí, segue que,

$$
I_{n}=\frac{2}{n !} \int_{0}^{\pi / 2}\left(\frac{\pi^{2}}{4}-t^{2}\right)^{n} \cos t d t=\frac{2}{n !}(\text { área sob o gráfico de } f)>0 .
$$


Lema 5.1 Se $r \in \mathbb{N}$, então $\lim _{n \rightarrow \infty} r^{n} I_{n}=0$.

Demonstração: Como $\frac{\pi^{2}}{4}-t^{2} \geq 0$ e $0 \leq \cos t \leq 1$ no intervalo $[0, \pi / 2]$ e, além disso $I_{n}>0$, então, $\left|r^{n} I_{n}\right|=r^{n} I_{n} \leq \frac{2 r^{n}}{n !} \int_{0}^{\pi / 2}\left(\frac{\pi^{2}}{4}-t^{2}\right)^{n} d t \leq \frac{2 r^{n}}{n !} \int_{0}^{\pi / 2}\left(\frac{\pi^{2}}{4}\right)^{n} d t=\frac{2 r^{n}}{n !}\left(\frac{\pi^{2}}{4}\right)^{n} \frac{\pi}{2}=\pi \frac{\left(\frac{r \pi^{2}}{4}\right)^{n}}{n !}$, isto é, $-\pi \frac{\left(\frac{r \pi^{2}}{4}\right)^{n}}{n !} \leq r^{n} I_{n} \leq \pi \frac{\left(\frac{r \pi^{2}}{4}\right)^{n}}{n !}$. Assim, conclui-se então do Exemplo 4.8 e do Teorema 4.1 que $\lim _{n \rightarrow \infty} r^{n} I_{n}=0$.

Lema 5.2 Se $\alpha$ é irracional e $\beta$ é racional não nulo, então $\alpha \beta$ é irracional.

Demonstração: Suponha que $\alpha \beta$ é racional. Como $\beta$ é racional não nulo, então $1 / \beta$ é racional. Daí, segue que $\alpha \beta \cdot(1 / \beta)$ é racional, i.e., $\alpha$ é racional (contradição!).

Teorema 5.1 Para todo inteiro n não negativo, tem-se que,

$$
I_{n}=P_{n}\left(\pi^{2}\right)
$$

onde $P_{n}$ é um polinômio de grau $\leq n$ com coeficientes inteiros.

Demonstração: A argumentação usada aqui está fundamentada no princípio da indução (forte). Seja $S(n)$ a seguinte sentença aberta relativa ao inteiro não negativo $n$.

$$
S(n): I_{n}=P_{n}\left(\pi^{2}\right) \text {, onde } P_{n} \text { é um polinômio de grau } \leq n \text { com coeficientes inteiros. }
$$

Base de Indução $(n=0,1)$ : Para $n=0$ podemos verificar facilmente que

$$
I_{0}=\int_{-\pi / 2}^{\pi / 2} \cos t d t=2 \int_{0}^{\pi / 2} \cos t d t=2=P_{0}\left(\pi^{2}\right),
$$

onde $P_{0}(t)=2$ é um polinômio de grau $\leq 0$ com coeficientes inteiros, i.e., $S(0)$ é verdadeira. Usando a técnica da integração por partes, obtemos que,

$$
\begin{aligned}
I_{1} & =\int_{-\pi / 2}^{\pi / 2}\left(\frac{\pi^{2}}{4}-t^{2}\right) \cos t d t \\
& =\left[\left(\frac{\pi^{2}}{4}-t^{2}\right) \operatorname{sen} t\right]_{-\pi / 2}^{\pi / 2}-\int_{-\pi / 2}^{\pi / 2}-2 t \operatorname{sen} t d t \\
& =2 \int_{-\pi / 2}^{\pi / 2} t \operatorname{sen} t d t \\
& =2\left[(-t \cos t)_{-\pi / 2}^{\pi / 2}-\int_{-\pi / 2}^{\pi / 2}-\cos t d t\right] \\
& =2 \int_{-\pi / 2}^{\pi / 2} \cos t d t \\
& =4 \\
& =P_{1}\left(\pi^{2}\right)
\end{aligned}
$$


onde $P_{1}(t)=4$ é um polinômio de grau $\leq 1$ com coeficientes inteiros, isto é, $S(1)$ é verdadeira. Passo Indutivo: Seja $m \geq 1$ um inteiro fixado. Suponha que $S(j)$ é verdadeira para todo inteiro $j$ satisfazendo $0 \leq j \leq m$ (Hipótese de Indução). Usando novamente a técnica da integração por partes, obtém-se que,

$$
\begin{aligned}
I_{m+1} & =\frac{1}{(m+1) !} \int_{-\pi / 2}^{\pi / 2}\left(\frac{\pi^{2}}{4}-t^{2}\right)^{m+1} \cos t d t \\
& =\frac{1}{(m+1) !}\left\{\left[\left(\frac{\pi^{2}}{4}-t^{2}\right)^{m+1} \operatorname{sen} t\right]_{-\pi / 2}^{\pi / 2}-\int_{-\pi / 2}^{\pi / 2}-2(m+1) t\left(\frac{\pi^{2}}{4}-t^{2}\right)^{m} \operatorname{sen} t d t\right\} \\
& =\frac{2}{m !} \int_{-\pi / 2}^{\pi / 2} t\left(\frac{\pi^{2}}{4}-t^{2}\right)^{m} \operatorname{sen} t d t \\
& =\frac{2}{m !}\left\{\left[-t\left(\frac{\pi^{2}}{4}-t^{2}\right)^{m} \cos t\right]_{-\pi / 2}^{\pi / 2}+\int_{-\pi / 2}^{\pi / 2}(\cos t)\left[\left(\frac{\pi^{2}}{4}-t^{2}\right)^{m}-2 t^{2} m\left(\frac{\pi^{2}}{4}-t^{2}\right)^{m-1}\right] d t\right\} \\
& =\frac{2}{m !}\left\{\int_{-\pi / 2}^{\pi / 2}(\cos t)\left[\left(\frac{\pi^{2}}{4}-t^{2}\right)^{m}-2 t^{2} m\left(\frac{\pi^{2}}{4}-t^{2}\right)^{m-1}\right] d t\right\} .
\end{aligned}
$$

Observando que $-t^{2}=\left(\frac{\pi^{2}}{4}-t^{2}\right)-\frac{\pi^{2}}{4}$, podemos reescrever a última igualdade da seguinte forma:

$$
\begin{aligned}
I_{m+1} & =\frac{2}{m !}\left\{\int_{-\pi / 2}^{\pi / 2}(\cos t)\left[\left(\frac{\pi^{2}}{4}-t^{2}\right)^{m}+2 m\left(\left(\frac{\pi^{2}}{4}-t^{2}\right)-\frac{\pi^{2}}{4}\right)\left(\frac{\pi^{2}}{4}-t^{2}\right)^{m-1}\right] d t\right\} \\
& =\frac{2}{m !}\left\{\int_{-\pi / 2}^{\pi / 2}(\cos t)\left[(2 m+1)\left(\frac{\pi^{2}}{4}-t^{2}\right)^{m}-\frac{\pi^{2} m}{2}\left(\frac{\pi^{2}}{4}-t^{2}\right)^{m-1}\right] d t\right\} \\
& =(4 m+2) \frac{1}{m !} \int_{-\pi / 2}^{\pi / 2}\left(\frac{\pi^{2}}{4}-t^{2}\right)^{m} \cos t d t-\pi^{2} \frac{1}{(m-1) !} \int_{-\pi / 2}^{\pi / 2}\left(\frac{\pi^{2}}{4}-t^{2}\right)^{m-1} \cos t d t \\
& =(4 m+2) I_{m}-\pi^{2} I_{m-1} .
\end{aligned}
$$

Temos da hipótese de indução que,

$$
I_{m}=P_{m}\left(\pi^{2}\right)=a_{m}\left(\pi^{2}\right)^{m}+a_{m-1}\left(\pi^{2}\right)^{m-1}+\cdots+a_{1} \pi^{2}+a_{0}=a_{m}\left(\pi^{2}\right)^{m}+\sum_{i=0}^{m-1} a_{i}\left(\pi^{2}\right)^{i}
$$

$\mathrm{e}$

$$
I_{m-1}=Q_{m-1}\left(\pi^{2}\right)=b_{m-1}\left(\pi^{2}\right)^{m-1}+b_{m-2}\left(\pi^{2}\right)^{m-2}+\cdots+b_{1} \pi^{2}+b_{0}=\sum_{i=0}^{m-1} b_{i}\left(\pi^{2}\right)^{i},
$$

com $a_{j} \in \mathbb{Z}(j=0,1, \ldots, m)$ e $b_{i} \in \mathbb{Z}(i=0,1, \ldots, m-1)$. Daí, segue que, 


$$
\begin{aligned}
I_{m+1} & =(4 m+2) I_{m}-\pi^{2} I_{m-1} \\
& =(4 m+2)\left[a_{m}\left(\pi^{2}\right)^{m}+\sum_{i=0}^{m-1} a_{i}\left(\pi^{2}\right)^{i}\right]-\pi^{2} \sum_{i=0}^{m-1} b_{i}\left(\pi^{2}\right)^{i} \\
& =\left[(4 m+2) a_{m}\right]\left(\pi^{2}\right)^{m}+\sum_{i=0}^{m-1}\left[(4 m+2) a_{i}\right]\left(\pi^{2}\right)^{i}-\sum_{i=0}^{m-1} b_{i}\left(\pi^{2}\right)^{i+1} \\
& =\left[(4 m+2) a_{m}\right]\left(\pi^{2}\right)^{m}-b_{m-1}\left(\pi^{2}\right)^{m}+\sum_{i=1}^{m-1}\left[(4 m+2) a_{i}\right]\left(\pi^{2}\right)^{i}-\sum_{i=0}^{m-2} b_{i}\left(\pi^{2}\right)^{i+1}+(4 m+2) a_{0} \\
& =\left[(4 m+2) a_{m}-b_{m-1}\right]\left(\pi^{2}\right)^{m}+\sum_{i=1}^{m-1}\left[(4 m+2) a_{i}\right]\left(\pi^{2}\right)^{i}-\sum_{i=1}^{m-1} b_{i-1}\left(\pi^{2}\right)^{i}+(4 m+2) a_{0} \\
& =\left[(4 m+2) a_{m}-b_{m-1}\right]\left(\pi^{2}\right)^{m}+\sum_{i=1}^{m-1}\left[(4 m+2) a_{i}-b_{i-1}\right]\left(\pi^{2}\right)^{i}+(4 m+2) a_{0} \\
& =R_{m+1}\left(\pi^{2}\right)
\end{aligned}
$$

onde, $R_{m+1}(t)=\left[(4 m+2) a_{m}-b_{m-1}\right] t^{m}+\left[(4 m+2) a_{m-1}-b_{m-2}\right] t^{m-1}+\cdots+\left[(4 m+2) a_{2}-\right.$ $\left.b_{1}\right] t^{2}+\left[(4 m+2) a_{1}-b_{0}\right] t+(4 m+2) a_{0}$ é um polinômio de grau $\leq m+1$ com coeficientes inteiros, i.e., $S(m+1)$ é verdadeira. Portanto, segue do princípio da indução (forte) que $S(n)$ é verdadeira para todo inteiro $n \geq 0$.

Corolário $5.1 \pi^{2}$ é irracional.

Demonstração: Suponha que $\pi^{2}$ é racional. $\operatorname{Logo}, \pi^{2}=r / s$, com $r, s \in \mathbb{N}$. Daí, segue do Teorema 5.1 que

$$
s^{n} P_{n}(r / s)=s^{n} I_{n}
$$

para todo inteiro $n$ não negativo. Considere-se a sequência $\left\{x_{n}\right\}_{n \geq 0}$ definida por $x_{n}=s^{n} P_{n}(r / s)$. Como $I_{n}>0$ e $P_{n}$ é um polinômio de grau $\leq n$ com coeficientes inteiros, então $x_{n} \in \mathbb{N}$ para todo inteiro $n$ não negativo. Por outro lado, o Lema 5.1 nos garante também que $\lim _{n \rightarrow \infty} s^{n} I_{n}=0$. Assim, concluímos então que $\left\{x_{n}\right\}_{n \geq 0}$ é uma sequência de inteiros positivos tal que $\lim _{n \rightarrow \infty} x_{n}=0$, o que contradiz o Lema 4.1.

Corolário $5.2 \pi$ é irracional.

Demonstração: Suponha que $\pi$ é racional. Então, $1 / \pi$ é racional não nulo. Mas, o Corolário 5.1 também nos garante que $\pi^{2}$ é irracional. Daí, segue do Lema 5.2 que $(1 / \pi) \cdot \pi^{2}$ é irracional, i.e., $\pi$ é irracional (contradição!).

\section{Conclusão}

A descoberta de novas demonstrações para um mesmo resultado é algo comum e que fascina muito os matemáticos. Obter provas mais simples e elegantes é sempre um prazeroso desafio.

Uma prova mais simples e direta do Corolário 5.2 a partir do Corolário 5.1, é a seguinte: Suponha que $\pi$ é racional. Então, $\pi \cdot \pi$ é racional, i.e., $\pi^{2}$ é racional, o que contradiz o Corolário 5.1 . 
Um dos principais exemplos dessa pluralidade de provas é o famoso teorema de Pitágoras, para o qual sabemos existirem diversas demonstrações. Pelo o que foi produzido acima, concluímos que também é possível provar a irracionalidade de $\pi$ sem o uso de ferramentas sofisticadas. Usando somente indução forte, integração por partes e conhecimentos básicos sobre sequências é possível provar a irracionalidade de $\pi^{2}$ (e portanto, de $\pi$ ).

Outro resultado sobre irracionalidade que também pode ser provado de forma elementar a partir de uma sequência $\left\{J_{n}\right\}_{n \geq 0}$ é a irracionalidade dos valores da exponencial em pontos racionais não nulos (como fazer isso?).

\section{Referências Bibliográficas}

[1] AIGNER, M.; ZIEGLER, G. M. Proofs from the book. 6th ed. Berlin: Springer-Verlag, 2018.

[2] BERGGREN, L.; BORWEIN, J.; BORWEIN, P. (Ed.). Pi: a source book. 3rd ed. New York: Springer-Verlag, 2004.

[3] BREUSCH, R. A proof of the irrationality of $\pi$. The American Mathematical Monthly, v. 61, n. 9, p. 631-632. 1954.

[4] DESBROW, D. On the irrationality of $\pi^{2}$. The American Mathematical Monthly, v. 97, n. 10, p. 903-906. 1990.

[5] GUNDERSON, D. S. Handbook of mathematical induction: theory and applications. Boca Raton: Chapman and Hall/CRC, 2011.

[6] HANCL, J. A simple proof of the irrationality of $\pi^{4}$. The American Mathematical Monthly, v. 93, n. 5, p. 374-375. 1986.

[7] LAMBERT, J. H. Mémoire sur quelques propriétés remarquables des quantités transcendentes circulaires et logarithmiques. Histoire de l'Academie Royale des Sciences et BellesLettres (Année 1761), Berlin, v. 17, p. 265-322, 1767.

[8] LITTLE, C. H. C.; TEO, K. L.; VAN BRUNT, B. Real analysis via sequences and series. New York: Springer-Verlag, 2015.

[9] MAKAROV, B. M. et al. Selected problems in real analysis. Providence: American Mathematical Society, 1992.

[10] NIVEN, I. A simple proof that $\pi$ is irrational. Bulletin of the American Mathematical Society, v. 53, n. 6, p. 509, 1947.

[11] NIVEN, I. Irrational numbers. [Washington, DC?]: Mathematical Association of America, 1956.

[12] STEWART, J. Cálculo. 7. ed. São Paulo: Cengage Learning, 2013. v. 1. 